\title{
Inattivazione dell'ormone tiroideo nei tumori stromali gastrointestinali
}

\author{
Marco Centanni · Susanna Carlotta Del Duca
}

Pubblicato online: 16 settembre 2014

(C) Springer International Publishing AG 2014

\section{Commento a:}

Thyroid hormone inactivation in gastrointestinal stromal tumors.

M.A. Maynard, A. Marino-Enriquez, J.A. Fletcher, D.M. Dorfman, C.P. Raut, L. Yassa, C. Guo, Y. Wang, C. Dorfman, H.A. Feldman, M.C. Frates, H. Song, R.H. Jugo, T. Taguchi, J.M. Hershman, P.R. Larsen, S.A. Huang.

$$
\text { N Engl J Med (2014) 370(14):1327-1334 }
$$

L'utilizzo di farmaci inibitori delle tirosin-chinasi (TKI), oggi largamente impiegati anche nel trattamento dei tumori stromali gastrointestinali (GIST), è associato a un'aumentata incidenza di ipotiroidismo. L'origine dell'ipofunzione tiroidea sembra essere legata a diversi meccanismi farmacoindotti, tra cui una riduzione della vascolarizzazione ghiandolare, l'inibizione dell'uptake dello iodio e l'induzione della desiodasi di tipo 3 (D3). In questo case-report, presentato da diverse scuole endocrinologiche di Boston, viene descritto il caso di un paziente con una voluminosa massa tumorale addominale, la cui diagnosi istologica deponeva per GIST. Prima di intraprendere il trattamento con sunitinib veniva valutata la funzione tiroidea del paziente da cui emergeva un ipotiroidismo grave $(\mathrm{TSH}=149 \mathrm{mcU} / \mathrm{ml}$ ). Durante il follow-up per il trattamento con TKI, nonostante dosi di oltre $300 \mathrm{mcg}$ di levotiroxina, i livelli di TSH del paziente rimanevano elevati, mentre i valori di $\mathrm{T} 4 \mathrm{e} \mathrm{T} 3$ risultavano al di sotto della norma. Questa situazione clinica è stata già descritta in pazienti affetti da emangioma e sembrava essere in- dipendente dall'impiego dei TKI. Questa forma di ipotiroidismo sembra essere conseguente a un'iperespressione della D3, ossia dell'enzima deputato all'inattivazione delle iodotironine, da parte delle cellule tumorali. Si determina pertanto il quadro dell'ipotiroidismo da consumo, causato dall'accelerata degradazione degli ormoni circolanti che supera la capacità ormonosintetica della tiroide. Per testare questa ipotesi gli autori hanno valutato la concentrazione di reverse T3, che risultava molto elevata, e l'attività della D3 mediante cromatografia e analisi immunoistochimica con anticorpi anti-D3, sul tessuto tumorale asportato chirurgicamente. L'espressione della D3 nelle cellule GIST era paragonabile a quella presente nella placenta a termine, che costituisce il tessuto sano con la maggiore attività desiodasica inattivante, e veniva confermata nell' $82 \%$ di 28 campioni chirurgici di GIST. È stato provato inoltre l'aumento dei livelli di mRNA codificante per la D3 nelle linee cellulari GIST dopo l'esposizione a dosi terapeutiche di TKI.

In conclusione, i risultati dello studio dimostrano che nei GIST l'iperespressione della D3 determina un ipotiroidismo che può essere ulteriormente esacerbato in pazienti che intraprendono un trattamento con TKI. In questi pazienti è pertanto consigliato il monitoraggio della funzione tiroidea, segnatamente quando sia in previsione un successivo trattamento con TKI. Inoltre, una condizione di ipotiroidismo da consumo, che richiede dosi molto elevate di tiroxina per essere corretto, può essere una caratteristica metabolica di tipologie tumorali diverse e una condizione più frequente di quanto finora dimostrato.

M. Centanni (凶) · S.C. Del Duca

Dipartimento di Scienze e Biotecnologie Medico-Chirurgiche, UOC di Endocrinologia, "Sapienza" Università di Roma, Latina, Italia

e-mail: marco.centanni@uniroma1.it 